\title{
Calculation of Core Losses Under DC Bias and Harmonics Based on Jiles-Atherton Dynamic Hysteresis Model Combined with Finite Element Analysis
}

\author{
Yang $\mathrm{Li}^{1}$, Lihua $\mathrm{Zhu}^{1}$, and Jianguo $\mathrm{Zhu}^{2}$, Senior member, IEEE \\ ${ }^{1}$ Tianjin Key Laboratory of Advanced Electrical Engineering and Energy Technology, \\ Tianjin Polytechnic University, Tianjin, China, m13516237893@163.com \\ ${ }^{2}$ Faculty of Engineering and Information Technology, University of Technology Sydney, Australia
}

\begin{abstract}
This paper presents a method to calculate the core losses in $\mathrm{SiFe}$ laminations under magnetizations with $\mathrm{DC}$ bias and harmonics. DC bias is usually generated by the ground return current of high voltage direct current (HVDC) system intrude into the windings of neutral-grounded transformers, which leads to increase of harmonics and core loss. For accurate calculation of core losses under DC bias, the Jiles-Atherton (JA) dynamic hysteresis model is incorporated into the finite element method. The J-A dynamic hysteresis model is constructed by combining the traditional $\mathbf{J}$-A hysteresis model with the models of instantaneous eddy current and excess losses. To account for the DC bias, the J-A dynamic model was modified by adjusting the parameters of instantaneous excess loss model. The theoretical results are verified by the measured results by a Single-Sheet Tester (SST 500).
\end{abstract}

Index Terms-J-A dynamic model, FEM, core loss, DC bias, harmonics.

\section{INTRODUCTION}

Direct current (DC) bias is an abnormal working state of power transformers, which is generated by the DC flows through the windings of neutral-grounded transformers via the neutral point. The application of high voltage direct current (HVDC) transmission systems is one of the main reasons for this phenomenon [1]. With the DC penetrating into the windings, the transformer is tending towards halfcycle saturation, distorting the magnetization waveform. Some experimental study shows that both the odd and even harmonic components are increased significantly under DC bias [2], leading to the increase of core loss and subsequent overheating. Therefore, the core loss is an important parameter for performance simulation. In general, the core loss under sinusoidal magnetizations can be empirically determined by the Steinmentz law, but the core loss under magnetizations with DC bias and harmonics become much more difficult to calculate [3]. In 1978, Newbury predicted the core loss for harmonic distortions [3], this method is based on the concept of separating the core loss into frequency independent and dependent parts, but no test on DC bias was mentioned. Thus, a hysteresis model which is able to calculate the dynamic core losses under various excitations in numerical simulation would be useful for engineering application.

In [4], the dynamic hysteresis model is derived to estimate the core losses but the model was not combined with any field analysis method so that the loss distribution and subsequent local overheating cannot be obtained. A core loss prediction method based on the dynamic Preisach model was proposed in [5], where the influence of DC bias was considered. It was claimed that this method was fast and simple for core loss calculation. However, it may be argued that this model is not simple to implement because the experimental data needed are numerous and delicate to identify.

This paper presents a method to calculate the core losses under magnetizations with DC bias and harmonics by the finite element method (FEM) incorporated with the JilesAtherton (J-A) dynamic model, and the calculation accuracy is verified by a comparison between calculated and measured results.

\section{DERIVATION OF THE J-A DYNAMIC HYSTERESIS EQUATION}

In a magnetic core, the total core loss can be separated into hysteresis, classical eddy current and excess losses. The classical eddy current loss is proportional to the square of the time derivative of flux density [6], and for a laminated core, it can be expressed as

$$
p_{e}=\frac{e^{2} \sigma}{2 \beta}\left(\frac{d B}{d t}\right)^{2}
$$

where $e$ is the thickness of material, $\sigma$ the conductivity of material, $\beta$ the geometric coefficient.

Due to the existence of magnetic domains, the local eddy currents are generated near the domain walls when the domain configuration changes under a dynamic excitation, resulting in the excess loss.

According to Bertotti's statistical theory of losses [7, 8], the excess loss can be calculated by

$$
p_{e x}=\frac{n_{0} V_{0}}{2}\left(\sqrt{1+\frac{4 \sigma G S V_{0}}{\left(n_{0} V_{0}\right)^{2}}\left|\frac{d B}{d t}\right|}-1\right)\left|\frac{d B}{d t}\right|
$$

where $n_{0}$ is the effective number of active magnetic objects (MOs) when the excitation is DC, $S$ the cross-sectional area of steel sheet, $G$ the coupling constant without unit, and $V_{0}$ the Statistical coupling field parameter, determining the ability of applied field to increase $n_{0}$ with increasing frequency. From the assumption that

$$
\sqrt{1+\frac{4 \sigma G S V_{0}}{\left(n_{0} V_{0}\right)^{2}}\left|\frac{d B}{d t}\right|}>>1
$$

(2) can be reduced as 


$$
p_{e x}=\left(G S V_{0} \sigma\right)^{1 / 2}\left|\frac{d B}{d t}\right|^{3 / 2}
$$

The J-A dynamic hysteresis model is constructed by combining the traditional J-A hysteresis model with the models of instantaneous eddy current and excess losses. Beginning from the traditional J-A model, two important equations are included in the traditional J-A model.

The anhysteretic magnetization equation can be expressed as

$$
M_{a n}=M_{s}\left[\operatorname{coth}\left(\frac{H+\alpha M_{a n}}{a}\right)-\left(\frac{a}{H+\alpha M_{a n}}\right)\right]
$$

where $M_{s}$ is the saturation magnetization, $a$ the loop shape parameter, and $\alpha$ the local field parameter.

The energy balance process in a magnetization can be expressed as

$$
\mu_{0} \int M d H_{e}=\mu_{0} \int M_{a n} d H_{e}-\mu_{0} k \delta \int \frac{d M_{i r r}}{d H_{e}} d H_{e}
$$

where $H_{e}$ is the effective field, $H_{e}=H+\alpha M, \delta$ the direction coefficient, $\delta=1$ for $d H / d t>0$, and $\delta=-1$ for $d H / d t<0$, and $k$ the pinning coefficient.The term of left side represents the actual magnetostatic energy, the right side represents the energy input and energy lost to pinning effect.

To take the eddy current and excess losses into consideration, the energy conservation formula of the traditional J-A model can be modified as

$$
\begin{aligned}
\mu_{0} \int M d H_{e}= & \mu_{0} \int M_{a n} d H_{e}-\mu_{0} k \delta \int \frac{d M_{i r r}}{d H_{e}} d H_{e}- \\
& \int \frac{e^{2} \sigma}{2 \beta}\left(\frac{d B}{d t}\right)^{2} d t-\int\left(G S V_{0} \sigma\right)^{1 / 2}\left|\frac{d B}{d t}\right|^{3 / 2} d t
\end{aligned}
$$

where the terms of right hand side mean that the input energy minus the hysteresis, eddy current and excess losses.

Because $H_{\mathrm{e}}=H+\alpha M$ and $B=\mu_{0}(H+M)$, the differential expression of the dynamic J-A model can be obtained as

$$
\frac{d M}{d B}=\frac{M-M_{a n}-\frac{c k \delta}{1-c} \frac{d M_{a n}}{d H_{e}}+k_{e} \frac{d B}{d t}+k_{e x} \delta\left|\frac{d B}{d t}\right|^{\frac{1}{2}}}{\mu_{0}(\alpha-1)\left(M_{a n}-M+\frac{c k \delta}{1-c} \frac{d M_{a n}}{d H_{e}}\right)-\frac{\mu_{0} k \delta}{1-c}}
$$

where $c$ is domain the rotation loss parameter, $k_{e}=e^{2} \sigma / 2 \beta$, and $k_{e x}=\left(G S V_{0} \sigma\right)^{1 / 2}$. Both $k_{e}$ and $k_{e x}$ can be deduced from the models of instantaneous eddy current and excess losses.

The measured data of Single-Sheet Tester (SST) are used to validate the improvement of J-A dynamic model compared with traditional J-A model. The major loops at $50 \mathrm{~Hz}$ predicted by the dynamic and traditional model are shown in Fig.1. together with the measured loop. The comparison showed that J-A dynamic model is more accurate than the traditional model under AC excitation, because the traditional J-A model cannot consider the influence of eddy current and excess losses on the magnetization process of materials.

In order to incorporate the J-A dynamic model into field analysis method, the differential expression of FEM can be written as

$$
\begin{aligned}
& \Omega: \nabla \times \frac{1}{\mu_{0}} \nabla \times \mathbf{A}-\nabla \times \mathbf{M}=\mathbf{J} \\
& \Gamma_{1}: \mathbf{A}=0 \\
& \Gamma_{2}: \frac{1}{\mu} \nabla \times \mathbf{A} \times \mathbf{n}=0
\end{aligned}
$$

where $\mathbf{A}$ is the vectorial magnetic potential, $\mu_{0}$ the permeability of vacuum, and $\mathbf{n}$ the unit normal vector. To incorporate the J-A dynamic hysteresis into FEM, the equation of magnetic field considering hysteresis is expressed as

$$
[\boldsymbol{K}]\left[\boldsymbol{A}_{n}\right]+\left[\boldsymbol{M}\left(\boldsymbol{A}_{n-1}\right)\right]=[\boldsymbol{F}]
$$

where $[\mathbf{M}]$ is the magnetization term obtain by $\mathrm{J}$-A dynamic model. Fig.2 illustrates a flow chart of the computational process.

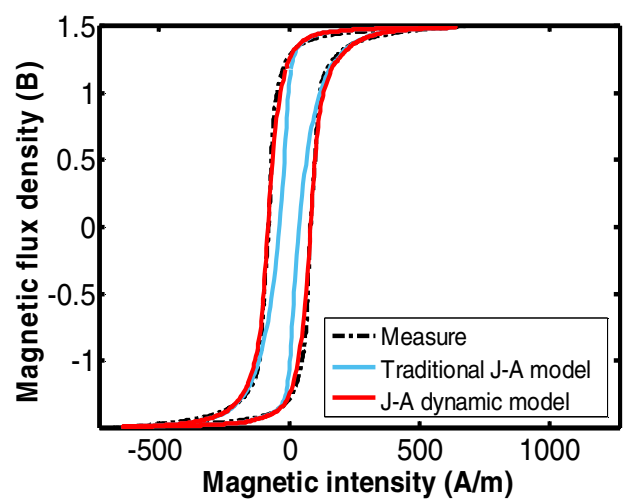

Fig. 1. Comparison of measured and predicted major loops.

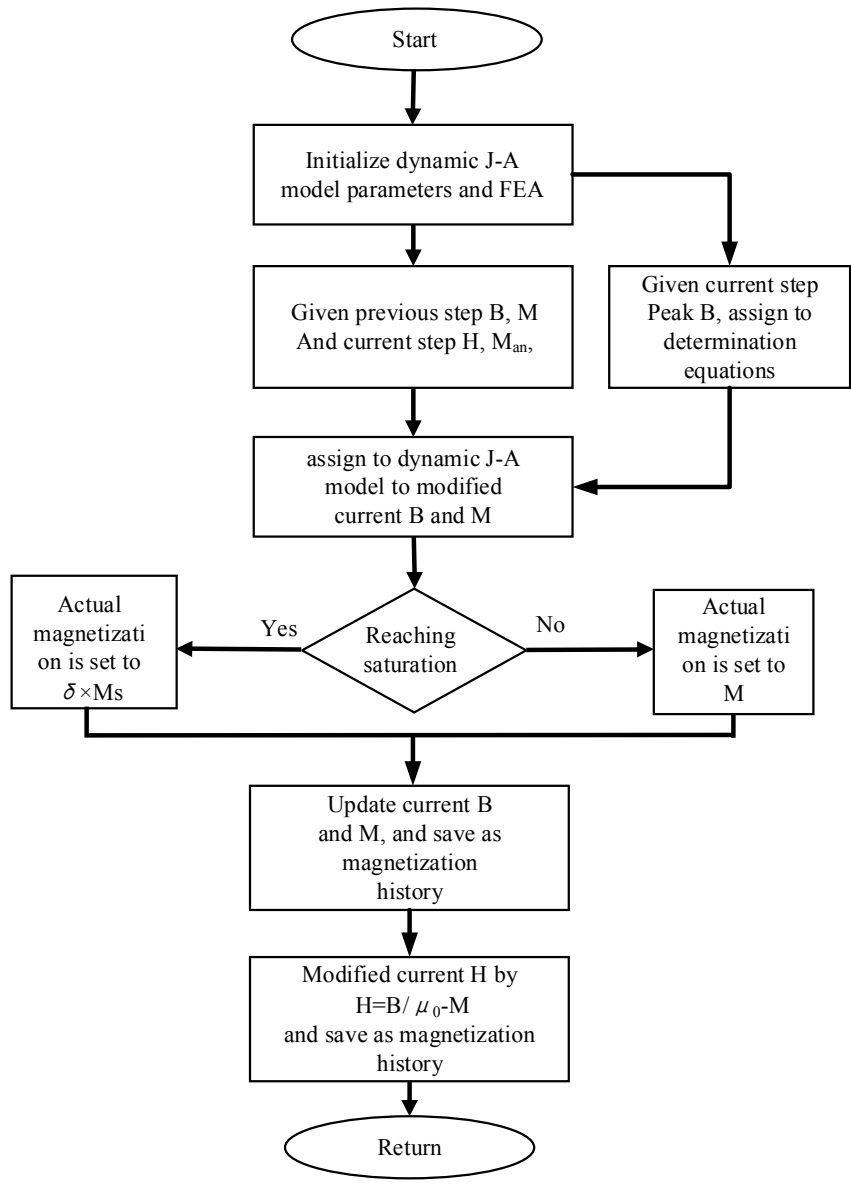

Fig. 2. flow chart of the computational process. 


\section{EXPERIMENTAL AND THEORETICAL ANALYSIS}

For this analysis, measurements under DC bias and harmonics have been carried out by a single sheet tester (SST 500) as shown in Fig.3. The B-H loops and the corresponding core losses of non-oriented SiFe laminations (50ww470, $600 \times 100 \times 0.5 \mathrm{~mm}$ ) were measured. The hysteresis loops were measured by uniformly-varying flux method, where the input current is varied in a way as to keep $d B / d t$ constant, such that the global eddy current loss is kept constant.

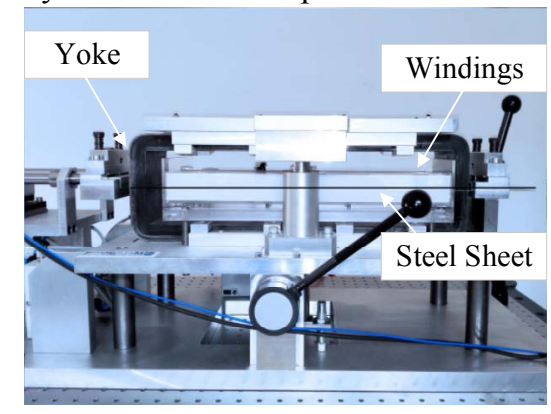

Fig.3. Single-Sheet Tester (SST 500).

However, the influence of DC bias on the excess and hysteresis losses cannot be neglected [8,9]. Because the excess loss is generated by the localized eddy currents ensuing from the domain wall motion, and the DC flux density will affect the domain distribution. In a similar way, the hysteresis loss is affected by the DC bias filed. Thus, the DC bias field must be taken into account.

Due to the J-A dynamic model is able to consider the influence of DC bias on hysteresis loss component, the excess loss component estimation becomes a special issue. To calculate the excess accurately, a method to modify the excess loss parameter $k_{\text {ex }}$ of excess loss model under DC bias is proposed.

The parameter $k_{\mathrm{ex}}$ can be calculated by $k_{\mathrm{ex}}=\left(G S V_{0} \sigma\right)^{1 / 2}$. It stressed that the $\mathrm{V}_{0}$ depend on the peak flux density and DC bias field [6]. Since the other parameters in $k_{\mathrm{ex}}$ are constant, different $\mathrm{V}_{0}$ leads to different $k_{\text {ex }}$. Thus, it's only necessary to identified the relationship between parameter $k_{\mathrm{ex}}$ and the input flux density and DC bias field.

To find the robust best fit, the parameter $k_{\mathrm{ex}}$ corresponding to different flux densities and DC bias fields are identified by the optimization algorithm. The error function of the algorithm gives

$$
F=\sqrt{\frac{\sum_{n=1}^{N}\left(1-B_{\mathrm{m} n} / B_{\mathrm{c} n}\right)}{N-1}}
$$

where $\mathrm{N}$ is the number of data points of hysteresis loop, $B_{\mathrm{m}}$ the measured data, and $B_{\mathrm{c}}$ the calculated data obtained by JA dynamic model with different $k_{\text {ex }}$.

When the error function satisfies the required accuracy, the distribution of $k_{\mathrm{ex}}$ corresponding to different flux densities and DC bias fields are obtained by parameter fitting method. The identified results are illustrated in Fig.4, and the results can be expressed approximately by a polynomial as

$$
k_{e x}=p_{1}+p_{2} H_{d}+p_{3} B+p_{4} H_{d} B+p_{5} B^{2}
$$

The other 6 parameters of the J-A dynamic model $(M \mathrm{~s}, \alpha$, $a, k, c, k_{e}$ ) were obtained in our previous work [10].

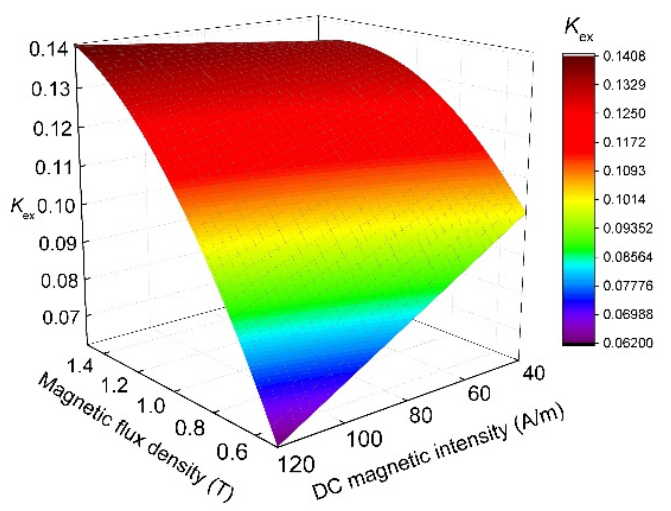

Fig. 4. The distribution of $\boldsymbol{k}_{\mathrm{ex}}$

\section{CAlculated Results AND VerificAtion}

With the adjusted parameter $k_{\text {ex }}$ obtained by (13), the hysteresis loops of non-oriented $\mathrm{SiFe}$ steel laminations were simulated and compared with the loops measured by an SST. Fig.5 shows the finite element model of SST, and Figs.6-8 compares the measured and calculated dynamic major loops with different input flux densities of $0.5 \mathrm{~T}, 1.0 \mathrm{~T}$ and $1.5 \mathrm{~T}$, where the measured results obtained under magnetizations comprised of a DC bias of $40 \mathrm{~A} / \mathrm{m}, 80 \mathrm{~A} / \mathrm{m}$ and $120 \mathrm{~A} / \mathrm{m}$, respectively. More detailed results are listed in Table I-III. The percentage error on total core loss is less than about $5 \%$. The data indicate that the core loss increases with the increase of the DC bias field at the input flux density at $0.5 \mathrm{~T}$ and 1.0T. However, the core loss remains unchanged with different DC bias fields when the input flux density is $1.5 \mathrm{~T}$, partly because the material almost reached saturation. Compared with the saturation magnetic fields, the DC bias fields have little influence on the magnetization process. For an increasing DC bias field, the excess loss shows a downward trend. These changes accord with magnetization theory and prove that the proposed model is effective.

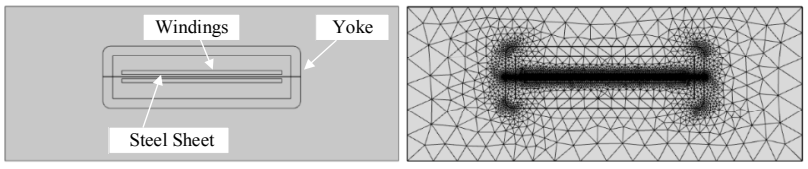

Fig.5. Finite element model of SST 500.

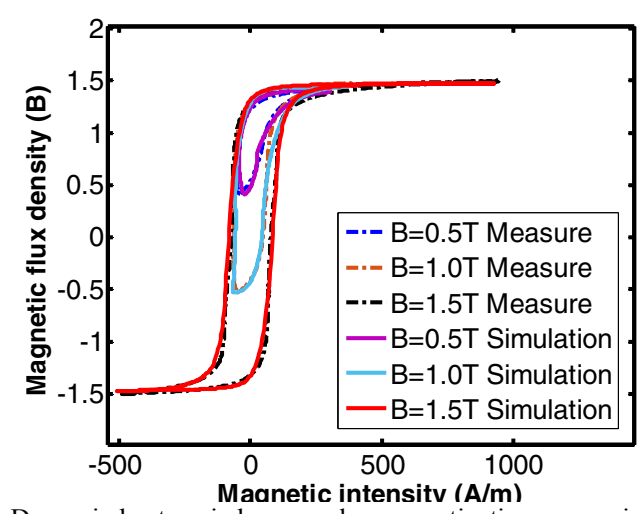

Fig.6. Dynamic hysteresis loops under magnetizations comprised of a DC bias of $40 \mathrm{~A} / \mathrm{m}$ with different peak flux densities.

where $p_{\mathrm{i}}(i=1-5)$ are constant coefficients, $H_{\mathrm{d}}$ the DC bias field, and $\mathrm{B}$ the peak flux density. 


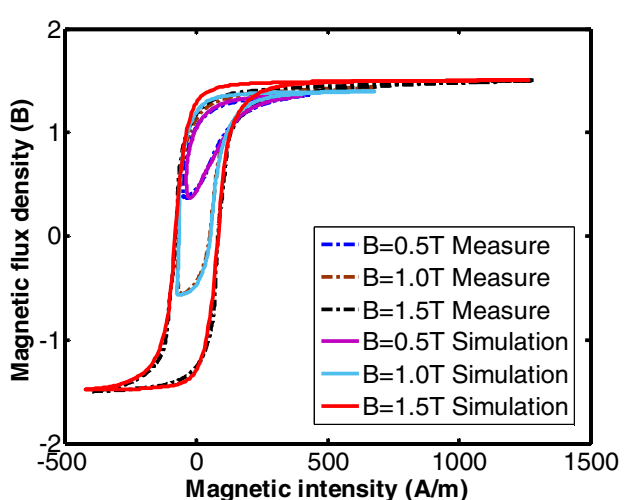

Fig.7. Dynamic hysteresis loops under magnetizations comprised of a DC bias of $80 \mathrm{~A} / \mathrm{m}$ with different peak flux densities.

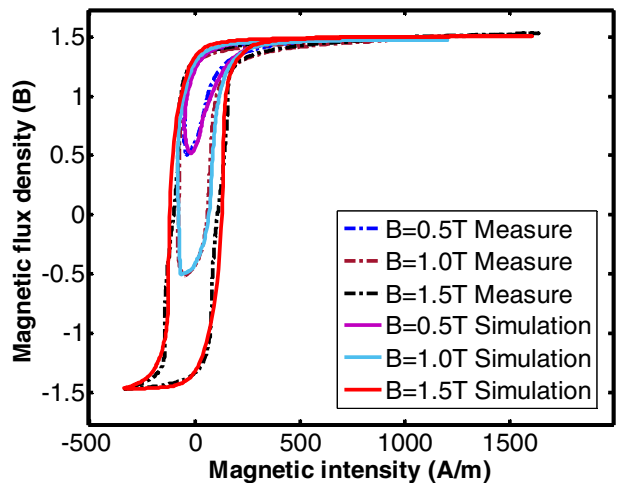

Fig.8. Dynamic hysteresis loops under magnetizations comprised of a DC bias of $120 \mathrm{~A} / \mathrm{m}$ with different peak flux densities.

TABLE I

COMPARASION OF CALCULATED AND MEASURED RESULTS UNDER DC BIAS FIELD 40A/m

\begin{tabular}{|c|c|c|c|c|c|}
\hline B (T) & $\begin{array}{l}\text { Calculated } \\
\text { Core loss } \\
(\mathrm{W} / \mathrm{kg})\end{array}$ & $\begin{array}{l}\text { Measured } \\
\text { Core loss } \\
(\mathrm{W} / \mathrm{kg})\end{array}$ & $\begin{array}{l}\text { Error } \\
(\%)\end{array}$ & $\begin{array}{l}\text { Calculated } \\
\text { Eddy } \\
\text { current } \\
\text { Loss }(\mathrm{W} / \mathrm{kg})\end{array}$ & $\begin{array}{c}\text { Calculated } \\
\text { Excess } \\
\text { loss } \\
(\mathrm{W} / \mathrm{kg})\end{array}$ \\
\hline 0.50 & 0.5434 & 0.5398 & -0.66 & 0.1798 & 0.0286 \\
\hline 1.00 & 1.4962 & 1.4523 & 1.72 & 0.4990 & 0.1108 \\
\hline 1.50 & 3.1217 & 3.1117 & -0.13 & 0.6902 & 0.2975 \\
\hline
\end{tabular}

TABLE II

COMPARASION OF CALCULATED AND MEASURED RESULTS UNDER DC BIAS FIELD 80A/m

\begin{tabular}{|c|c|c|c|c|c|}
\hline B (T) & $\begin{array}{l}\text { Calculated } \\
\text { Core loss } \\
(\mathrm{W} / \mathrm{kg})\end{array}$ & $\begin{array}{l}\text { Measured } \\
\text { Core loss } \\
(\mathrm{W} / \mathrm{kg})\end{array}$ & $\begin{array}{l}\text { Error } \\
(\%)\end{array}$ & $\begin{array}{l}\text { Calculated } \\
\text { Eddy } \\
\text { current } \\
\text { Loss }(\mathrm{W} / \mathrm{kg})\end{array}$ & $\begin{array}{c}\text { Calculated } \\
\begin{array}{c}\text { Excess } \\
\text { loss } \\
(\mathrm{W} / \mathrm{kg})\end{array}\end{array}$ \\
\hline 0.50 & 0.6416 & 0.6488 & -0.11 & 0.1800 & 0.0260 \\
\hline 1.00 & 1.5846 & 1.5182 & -4.38 & 0.5527 & 0.1039 \\
\hline 1.50 & 3.1695 & 3.1052 & -2.07 & 0.7710 & 0.2973 \\
\hline
\end{tabular}

TABLE III

COMPARASION OF CALCULATED AND MEASURED RESULTS UNDER DC BIAS FIELD 120A/m

\begin{tabular}{|c|c|c|c|c|c|}
\hline B (T) & $\begin{array}{l}\text { Calculated } \\
\text { Core loss } \\
(\mathrm{W} / \mathrm{kg})\end{array}$ & $\begin{array}{l}\text { Measured } \\
\text { Core loss } \\
(\mathrm{W} / \mathrm{kg})\end{array}$ & $\begin{array}{l}\text { Error } \\
(\%)\end{array}$ & $\begin{array}{l}\text { Calculated } \\
\text { Eddy } \\
\text { current } \\
\text { Loss }(\mathrm{W} / \mathrm{kg})\end{array}$ & $\begin{array}{c}\text { Calculated } \\
\text { Excess } \\
\text { loss } \\
(\mathrm{W} / \mathrm{kg})\end{array}$ \\
\hline 0.50 & 0.6693 & 0.6601 & -1.39 & 0.2309 & 0.0256 \\
\hline 1.00 & 1.6017 & 1.5640 & -2.40 & 0.5398 & 0.1005 \\
\hline 1.50 & 3.0743 & 3.1149 & -1.30 & 0.6451 & 0.3009 \\
\hline
\end{tabular}

Since the harmonic components would increase significantly under DC bias, the implementation of the model is tested under the effect of harmonics and DC bias. Fig.9 depicts the calculated and measured dynamic hysteresis loops when the excitation consists of a second order harmonic component $(100 \mathrm{~Hz})$ and a DC bias of $80 \mathrm{~A} / \mathrm{m}$, and Fig. 10 the comparasion under magnetizations comprised of a third order harmonic component $(150 \mathrm{~Hz})$ and a DC bias of $80 \mathrm{~A} / \mathrm{m}$. A large discrepancy occurs when the input flux density at $0.5 \mathrm{~T}$ under a DC bias and third order harmonic. Although the error on core loss is still close, there is a distortion in the minor loops. It was found that the discrepancy of simulated dynamic hysteresis loops increased in such case, and it was most probably caused by the more complex magnetization process, but the calculated and measured core losses are close as shown in Tables IV and V.

Compared with the case in which only DC bias exists, the core loss increases a lot when the magntizations comprised DC bias and harmonics. It was found that all three components of core losses increased, and thus it could be concluded that the loss caused by harmonics account for a large part of the core loss increment under DC bias condition when the bias field is between $40 \mathrm{~A} / \mathrm{m}-120 \mathrm{~A} / \mathrm{m}$.

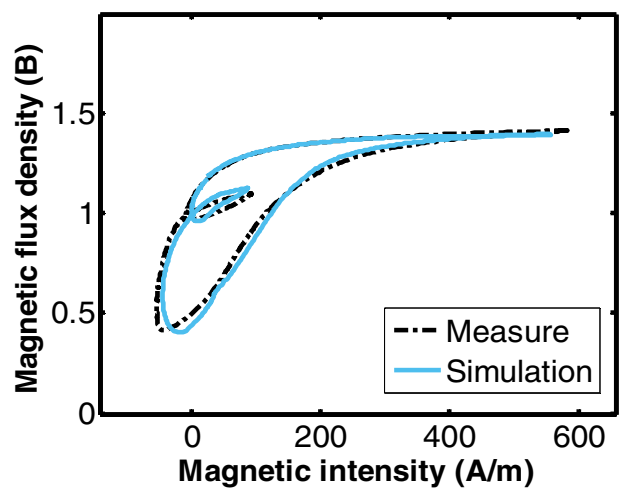

(a) The input peak flux density is $0.5 \mathrm{~T}$

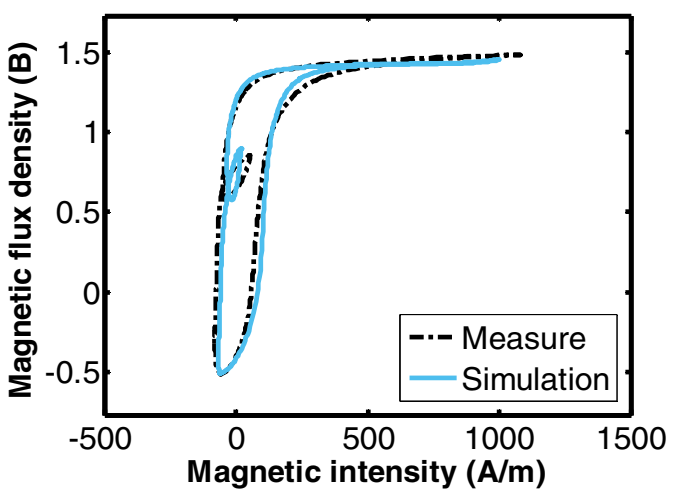

(b) The input peak flux density is $1.0 \mathrm{~T}$

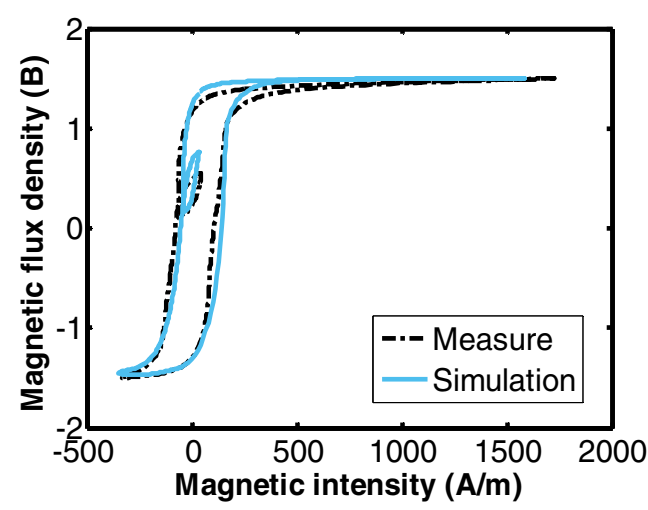

(c) The input peak flux density is $1.5 \mathrm{~T}$ 
Fig.9. Dynamic hysteresis loops under magnetizations comprised of a DC bias of $80 \mathrm{~A} / \mathrm{m}$ and a second order harmonic component and at different input peak flux densities

TABLE IV

COMPARASION OF CALCULATED AND MEASURED RESULTS

\begin{tabular}{|c|c|c|c|c|c|}
\hline $\mathrm{B}(\mathrm{T})$ & $\begin{array}{l}\text { Calculated } \\
\text { Core loss } \\
(\mathrm{W} / \mathrm{kg})\end{array}$ & $\begin{array}{l}\text { Measured } \\
\text { Core loss } \\
(\mathrm{W} / \mathrm{kg})\end{array}$ & $\begin{array}{l}\text { Error } \\
(\%)\end{array}$ & $\begin{array}{l}\text { Calculated } \\
\text { Eddy } \\
\text { current } \\
\text { Loss }(\mathrm{W} / \mathrm{kg})\end{array}$ & $\begin{array}{c}\text { Calculated } \\
\text { Excess } \\
\text { loss } \\
(\mathrm{W} / \mathrm{kg})\end{array}$ \\
\hline 0.50 & 0.7745 & 0.74 & 3.61 & 0.2607 & 0.0758 \\
\hline 1.00 & 1.9683 & 1.8428 & 6.37 & 0.7503 & 0.1768 \\
\hline 1.50 & 3.8952 & 3.8872 & 0.21 & 1.4514 & 0.2925 \\
\hline
\end{tabular}

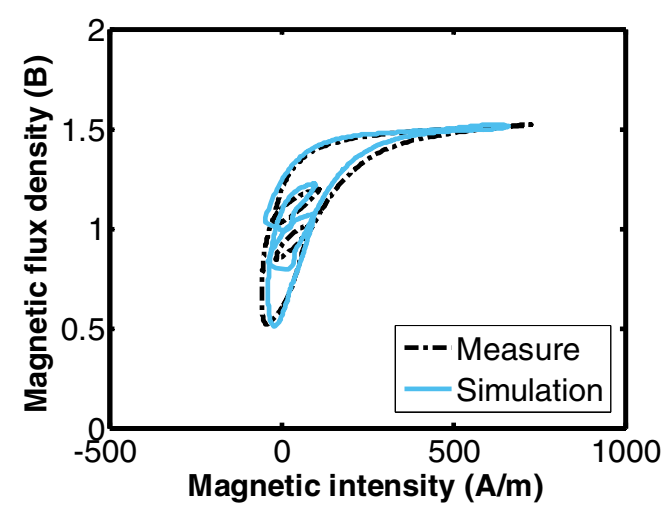

(a) The input peak flux density is $0.5 \mathrm{~T}$

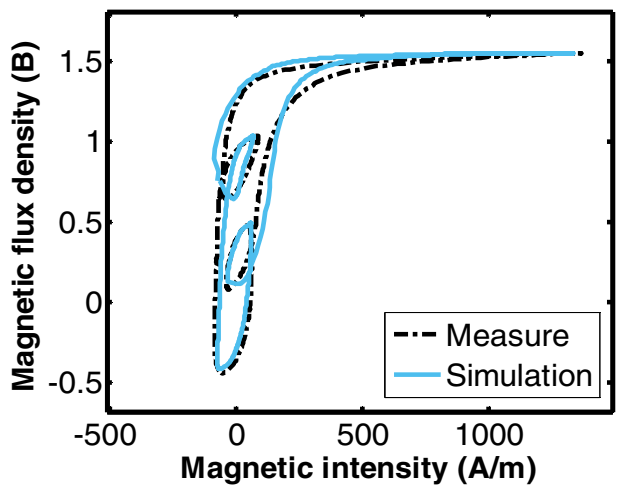

(b) The input peak flux density is $1.0 \mathrm{~T}$

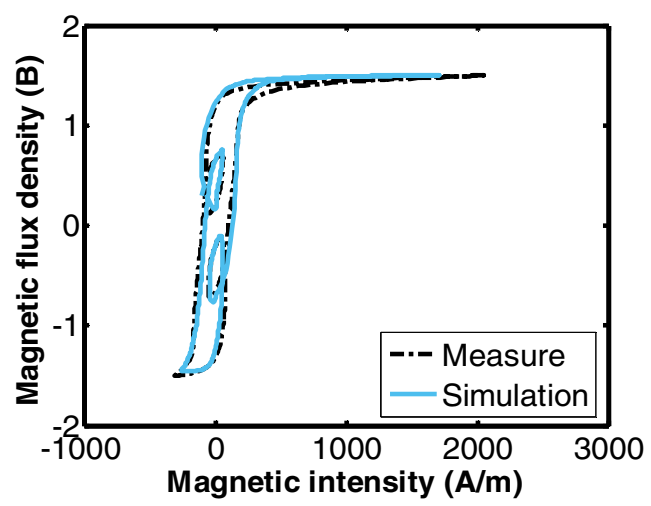

(c) The input peak flux density is $1.5 \mathrm{~T}$

Fig.10. Dynamic hysteresis loops under magnetizations comprised of a $\mathrm{DC}$ bias of $80 \mathrm{~A} / \mathrm{m}$ and a third order harmonic component and at different input peak flux densities

TABLE V

COMPARASION OF CALCULATED AND MEASURED RESULTS

\begin{tabular}{|c|c|c|c|c|c|}
\hline B (T) & $\begin{array}{l}\text { Calculated } \\
\text { Core loss } \\
(\mathrm{W} / \mathrm{kg})\end{array}$ & $\begin{array}{l}\text { Measured } \\
\text { Core loss } \\
(\mathrm{W} / \mathrm{kg})\end{array}$ & $\begin{array}{c}\text { Error } \\
(\%)\end{array}$ & $\begin{array}{l}\text { Calculated } \\
\text { Eddy current } \\
\text { Loss }(\mathrm{W} / \mathrm{kg})\end{array}$ & $\begin{array}{c}\text { Calculated } \\
\text { Excess } \\
\text { loss } \\
(\mathrm{W} / \mathrm{kg})\end{array}$ \\
\hline 0.50 & 0.9010 & 0.8986 & -0.27 & 0.2744 & 0.0881 \\
\hline
\end{tabular}

\begin{tabular}{|l|l|l|l|l|l|}
\hline 1.00 & 2.3150 & 2.3675 & 6.37 & 0.8420 & 0.2021 \\
\hline 1.50 & 4.5366 & 4.7434 & 4.36 & 1.8409 & 0.3707 \\
\hline
\end{tabular}

\section{CONCLUSION}

The core losses in $\mathrm{SiFe}$ laminations under magnetizations with DC bias and harmonics is calculated by incorporating the J-A dynamic model into the FEM. The proposed J-A dynamic model is constructed by combining the traditional $\mathrm{J}$ A hysteresis model with the models of instantaneous eddy current and excess losses. To consider the influences of DC bias on excess loss, a computational scheme is presented to identified the dynamic parameter.

An experimental verification of proposed model was given by comparing total core losses and dynamic hysteresis loops under magnetizations comprised of DC bias of $40 \mathrm{~A} / \mathrm{m}$, $80 \mathrm{~A} / \mathrm{m}$ and $120 \mathrm{~A} / \mathrm{m}$ with different flux densities. To further confirm the accuracy of proposed model, dynamic hysteresis loops and core losses under magnetizations comprised of a DC bias of $80 \mathrm{~A} / \mathrm{m}$ and harmonic components are used to compare with the experimental results.

The comparison shows that the J-A dynamic model is able to reflect the magnetization process occurring in $\mathrm{SiFe}$ laminations accurately, and the calculated and measured core losses are very close. It has been demonstrated that proposed computational method is sufficiently accurate for numerical simulation under various excitations. Combining with the FEM, the model is able to achieve optimum design and accurate performance prediction.

\section{ACKNOWLEDGEMENT}

This work was supported by the National Natural Science Foundation of China under Grant 51507110.

\section{REFERENCES}

[1] Zhicheng Xie, Xiangning Lin, and Zheyuan ZHANG, et al, "Advanced DC Bias Suppression Strategy based on Finite DC Blocking Devices"., unpublished.

[2] Z. Li, Q. Li, and C. Li, "Harmonic distortion feature of AC transformers caused by DC bias". in Power System Protection and Control, vol. no. 24, pp. 52-55, 2010.

[3] Roger A. Newbury, "Prediction of Loss in Silicon Steel from Distorted Waveforms". in IEEE Transactions on Magnetics, vol. MAG-14, no. 4, pp., 1978.

[4] Yang Wang, and Zhizhen Liu, "Estimation Model of Core Loss Under DC Bias". in IEEE Transactions on Applied Superconductivity, vol. 26, no. 7, pp. 1-5, 2016.

[5] Olivier de la Barriere, Carlo Ragusa, and Carlo Appino, et al, "Prediction of Energy Losses in Soft Magnetic Materials Under Arbitrary Induction Waveforms and DC Bias". in IEEE Transactions on Industrial Electronics, vol. 64, no. 3, pp. 2522-2529, 2017.

[6] E. Barbisio, O. Bottauscio, and M. Chiampi, et al, "Prediction of magnetic power losses in soft laminations under DC-biased supply". in Journal of Magnetism and Magnetic Materials, vol. 290-291, no. pp. 1476-1479, 2005.

[7] G. Bertotti, "Physical interpretation of eddy current losses in ferromagnetic materials. I. Theoretical considerations". in Journal of Applied Physics, vol. 57, no. 6, pp. 2110-2117, 1985.

[8] E. Barbisio, F. Fiorillo, and C. Ragusa, "Predicting Loss in Magnetic Steels Under Arbitrary Induction Waveform and With Minor Hysteresis Loops". in IEEE Transactions on Magnetics, vol. 40, no. 4, pp. 1810-1819, 2004.

[9] Edoardo Barbisio, Oriano Bottauscio, and Mario Chiampi, et al, "Analysis of AC magnetic properties in SiFe laminations under DCbiased magnetisation". in Physica B: Condensed Matter, vol. 343, no. $1-4$, pp. 127-131, 2004.

[10] Yang Li, Lihua Zhu, and Jianguo Zhu, "Core Loss Calculation Based on Finite Element Method with Jiles-Atherton Dynamic Hysteresis Model"., unpublished. 